\title{
Effect of impurity substitution on band structure and mass renormalization of the correlated $\mathrm{FeTe}_{0.5} \mathrm{Se}_{0.5}$ superconductor
}

\author{
S. Thirupathaiah,,${ }^{1,}$ J. Fink, ${ }^{2}$ P. K. Maheshwari, ${ }^{3}$ V. V. Ravi Kishore,,${ }^{1}$ Z.-H. Liu, ${ }^{2}$ E. D. L. Rienks, ${ }^{2}$ B. Büchner, ${ }^{2}$ \\ V. P. S. Awana, ${ }^{3}$ and D. D. Sarma ${ }^{1}$ \\ ${ }^{1}$ Solid State and Structural Chemistry Unit, Indian Institute of Science, Bangalore, Karnataka, 560012, India \\ ${ }^{2}$ Leibniz Institut für Festökrper- und Werkstoffforschung IFW Dresden, D-01171 Dresden, Germany \\ ${ }^{3}$ CSIR-National Physical Laboratory, New Delhi 110012, India \\ (Received 3 March 2016; revised manuscript received 12 April 2016; published 24 May 2016)
}

\begin{abstract}
Using angle-resolved photoemission spectroscopy (ARPES), we studied the effect of the impurity potential on the electronic structure of $\mathrm{FeTe}_{0.5} \mathrm{Se}_{0.5}$ superconductor by substituting $10 \%$ of $\mathrm{Ni}$ for Fe, which leads to an electron doping of the system. We could resolve three hole pockets near the zone center and an electron pocket near the zone corner in the case of $\mathrm{FeTe}_{0.5} \mathrm{Se}_{0.5}$, whereas only two hole pockets near the zone center and an electron pocket near the zone corner are resolved in the case of $\mathrm{Fe}_{0.9} \mathrm{Ni}_{0.1} \mathrm{Te}_{0.5} \mathrm{Se}_{0.5}$, suggesting that the hole pocket having predominantly the $x y$ orbital character is very sensitive to the impurity scattering. Upon electron doping, the size of the hole pockets decreases and the size of the electron pockets increases as compared to the host compound. However, the observed changes in the size of the electron and hole pockets are not consistent with the rigid-band model. Moreover, the effective mass of the hole pockets is reduced near the zone center and of the electron pockets is increased near the zone corner in the doped $\mathrm{Fe}_{0.9} \mathrm{Ni}_{0.1} \mathrm{Te}_{0.5} \mathrm{Se}_{0.5}$ as compared to $\mathrm{FeTe}_{0.5} \mathrm{Se}_{0.5}$. We refer these observations to the changes of the spectral function due to the effect of the impurity potential of the dopants.
\end{abstract}

DOI: 10.1103/PhysRevB.93.205143

\section{INTRODUCTION}

Most of the parent iron pnictides at ambient conditions [1-6], except LiFeAs $\left(T_{c} \approx 18 \mathrm{~K}\right)$ [7] and FeSe $\left(T_{c} \approx 8 \mathrm{~K}\right)$ [8], are antiferromagnetic metals and they show superconductivity upon chemical doping or substitution [9-12]. Therefore superconductivity in iron-based superconductors is very often induced by impurity substitution into the parent compound followed by suppressing the long-range antiferromagnetic ordering $[13,14]$. Hence a good knowledge on how the impurity dopants suppress the antiferromagntic ordering and lead the system to superconductivity is very crucial to understand the mechanism of high- $T_{c}$ superconductivity in iron-based superconductors.

Theoretical works suggest that impurities act as scattering centers, which lead to a broadening of the bands [15-18]. These studies also point out that with the $3 d$ transition element substitution for $\mathrm{Fe}$, a part of the additional electrons from the transition metal remain localized at the constituents. However, there are several ARPES reports that demonstrate that the Co substitution for Fe donates the charge carriers to the host system according to a rigid band model [19-23]. On the other hand, with the substitution of $\mathrm{Ni}$ and $\mathrm{Cu}$ for $\mathrm{Fe}$, the additional doping concentration is reduced, while for $\mathrm{Zn}$ the additional electrons are completely localized at the $\mathrm{Zn}$ ions [17,24]. In contradiction to the ARPES studies, a report using x-ray absorption spectroscopy suggested that the Co substitution for $\mathrm{Fe}$ atom is nothing but a kind of isovalent substitution [25]. Recent consensus is that upon substitution of the $3 d$ transition element for Fe (electron doping), the volume of the electron and hole Fermi surfaces increases and decreases, respectively,

\footnotetext{
*t.setti@sscu.iisc.ernet.in
}

qualitatively consistent with the rigid-band model, but the effective electron doping decreases in going from Co to $\mathrm{Zn}$ $[17,24]$. Similarly, the localization of the doped holes is noticed when $\mathrm{Fe}$ is replaced by $\mathrm{Cr}$ [26] or Mn [27].

Many ARPES studies, dealing with the effect of impurities on the electronic structure, are available on weakly correlated 122-type [20-23,28] and 111-type [29-31] iron pnictides. Intriguingly, till now, no ARPES study has been made on the more correlated charge doped 11-type iron chalcogenides, which motivated us for the present study, though there are transport [32-35], thermal [36], and magnetic measurements [37] reporting on this issue. These studies suggest that the transition metal substitution for $\mathrm{Fe}$ in $\mathrm{FeTe}_{1-x} \mathrm{Se}_{x}$ leads to a metal-insulator transition at high concentrations.

In this paper, we report on the electronic band structure, the Fermi surface topology and the spectral function analysis of the $\mathrm{Fe}_{1-x} \mathrm{Ni}_{x} \mathrm{Te}_{0.5} \mathrm{Se}_{0.5}$ compounds $(x=0$ and 0.1$)$ using ARPES, in order to understand the effect of $\mathrm{Ni}$ substitution on the electronic structure and the electronic correlations of $\mathrm{FeTe}_{0.5} \mathrm{Se}_{0.5}$ superconductor. We could resolve three hole pockets near the zone center and an electron pocket near the zone corner in the case of $\mathrm{FeTe}_{0.5} \mathrm{Se}_{0.5}$, consistent with the band structure of the similar compounds [38-43], whereas only two hole pockets are resolved near the center and an electron pocket is resolved near the corner of the Brillouin zone in the case of $\mathrm{Fe}_{0.9} \mathrm{Ni}_{0.1} \mathrm{Te}_{0.5} \mathrm{Se}_{0.5}$, suggesting that the hole pocket that has predominantly $x y$ orbital character is very sensitive to impurity scattering. We observe a decrease in the size of the hole pockets and an increase in the size of the electron pockets with $\mathrm{Ni}$ substitution, suggesting an effective electron doping. However, the observed change in the size of the electron and hole pockets is not consistent with the rigid band model. We further noticed that the mass renormalization is reduced near the zone center and increased 
(a)

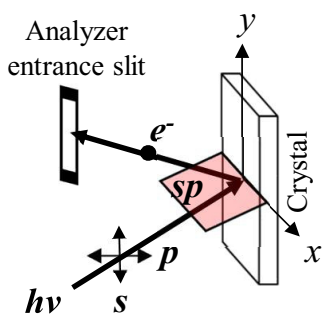

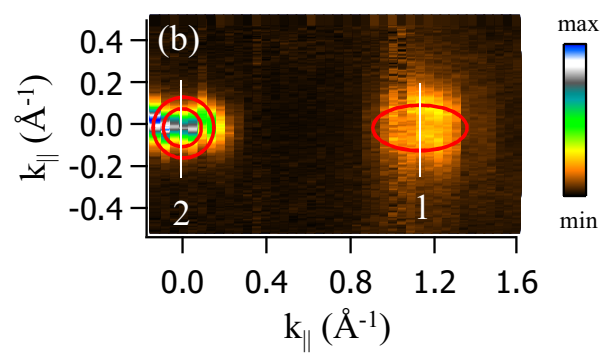
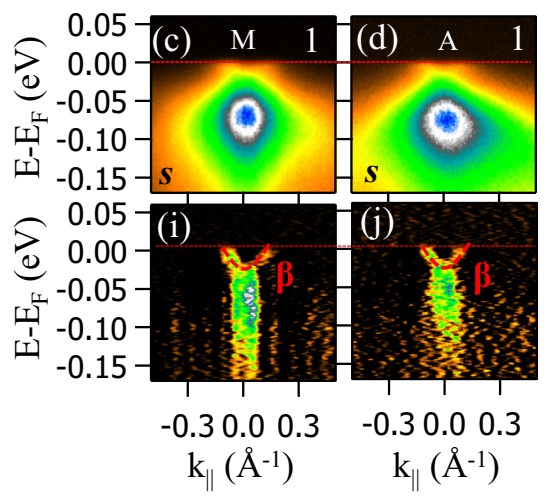
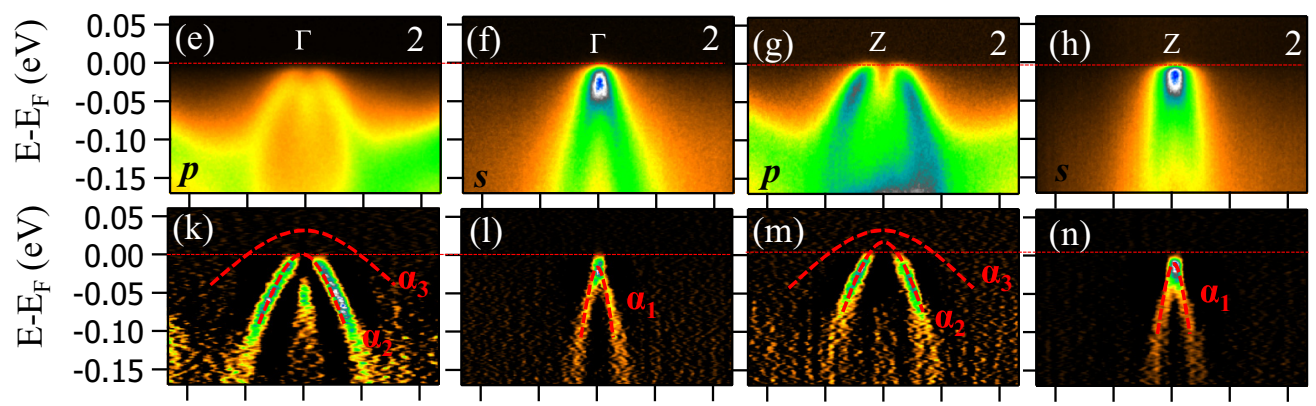

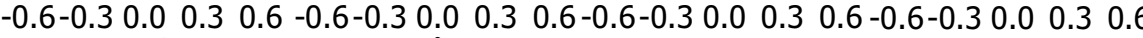

$\mathrm{k}_{\|}\left(\AA^{-1}\right)$
$\mathrm{k}_{\|}\left(\AA^{-1}\right)$
$\mathrm{k}_{\|}\left(\AA^{-1}\right)$

$\mathrm{k}_{\|}\left(\AA^{-1}\right)$

FIG. 1. ARPES data from $\mathrm{FeTe}_{0.5} \mathrm{Se}_{0.5}$. In (a), we schematically show our measuring geometry in which we define $s$ - and $p$-plane polarized lights with respect to the analyzer entrance slit. (b) Fermi surface map measured using $s$-polarized light with a photon energy $h v=81 \mathrm{eV}$. (c) and (d) show the energy distribution maps (EDMs) taken close to the high-symmetry points $M$ and $A$ (see text), respectively, measured using $s$-polarized light along cut 1 as showed on the FS map. (e) and (f) show EDMs taken near $\Gamma$ measured using $p$ - and $s$-polarized lights, respectively, along cut 2 as shown on the FS map. (g) and (h) are analogous data to (e) and (f), respectively, but taken near the $Z$ point. (i)-(n) are the second derivatives of (c)-(h). The red colored contours in (b) are guides to the eye schematically representing the Fermi sheets, and the curves in (i)-(n) are guides to the eye schematically representing the band dispersions. In (o) and (p), we show the renormalized DFT-LDA band structure (black curves) overlaid on the experimental (red curves) band dispersions. We have normalized the EDMs by higher orders of the monochromator above the Fermi level.

near the zone corner in $\mathrm{Fe}_{0 .} \mathrm{Ni}_{0.1} \mathrm{Te}_{0.5} \mathrm{Se}_{0.5}$ as compared to $\mathrm{FeTe}_{0.5} \mathrm{Se}_{0.5}$.

\section{EXPERIMENTAL DETAILS}

Single crystals of $\mathrm{Fe}_{1-x} \mathrm{Ni}_{x} \mathrm{Te}_{0.5} \mathrm{Se}_{0.5}(x=0$ and 0.1$)$ were grown at National Physical Laboratory in Delhi using self-flux. $\mathrm{FeTe}_{0.5} \mathrm{Se}_{0.5}$ shows superconducting transition at a $T_{c} \approx 14 \mathrm{~K}$, while $\mathrm{Fe}_{0.9} \mathrm{Ni}_{0.1} \mathrm{Te}_{0.5} \mathrm{Se}_{0.5}$ shows no superconductivity down to $2 \mathrm{~K}[44,45]$. ARPES measurements were carried out at BESSY II (Helmholtz Zentrum Berlin) synchrotron radiation facility at the UE112-PGM2b beam-line using the " 13 -ARPES" $[46,47]$ and the " 12 -ARPES" end stations equipped with SCIENTA R4000 analyzer and SCIENTA R8000 analyzer, respectively.

The measurements on $\mathrm{FeTe}_{0.5} \mathrm{Se}_{0.5}$ superconductors were measured at the " 13 -ARPES" end station. The total energy resolution was set between 5 and $10 \mathrm{meV}$, depending on the applied photon energy. Samples were cleaved in situ at a sample temperature lower than $20 \mathrm{~K}$. The measurements were carried out at a sample temperature $T \approx 1 \mathrm{~K}$. The measurements on $\mathrm{Fe}_{0.9} \mathrm{Ni}_{0.1} \mathrm{Te}_{0.5} \mathrm{Se}_{0.5}$ are measured at the " $1{ }^{2}$-ARPES" end station. The total energy resolution was set between 15 to $20 \mathrm{meV}$, depending on the applied photon energy. Samples were cleaved in situ and measured at a sample temperature of $T \approx 50 \mathrm{~K}$.

\section{CALCULATIONS}

To understand the experimental data, we have performed a theoretical analysis of the electronic band structure of FeTe. The band-structure calculations are done within the local density approximation (LDA) using the PAW pseudopotentials and the plane waves $[48,49]$ as implemented in the Vienna $a b$ initio simulation package (VASP) [50-53]. We used the experimental lattice constants, while the internal coordinates are freely relaxed. Monkhorst-Pack $k$-point mesh of $16 \times$ $16 \times 12$ has been used for the Brillouin zone sampling. The plane-wave cutoff energy was set at $400 \mathrm{eV}$.

\section{RESULTS}

Figure 1 shows ARPES data from the $\mathrm{FeTe}_{0.5} \mathrm{Se}_{0.5}$ superconductor. Figure 1(a) depicts our measuring geometry where we define the $s$ - and $p$-plane polarized lights with respect to the analyzer entrance slit. Figure 1(b) is the Fermi surface map measured using $s$-polarized light with an excitation energy $h v=81 \mathrm{eV}$. The data were recorded at a sample temperature 
of $1 \mathrm{~K}$. To avoid influence of the SC gap, the FS map is extracted by integrating over an energy window of $10 \mathrm{meV}$ centered at $E_{F}$, in which the hole pockets at the zone center and the electron pockets at the zone corner are seen. Figures 1(c) and 1(d) show energy distribution maps (EDMs) taken along the cut 1 as shown on the FS map measured using the $s$ polarized light with photon energies 58 and $45 \mathrm{eV}$, respectively. According to the equation, $k_{\perp}=\sqrt{\frac{2 m_{e}}{\hbar^{2}}\left[E_{\mathrm{kin}} \cos ^{2} \theta+V_{0}\right]}$ (the inner potential $V_{0}=15 \pm 3 \mathrm{eV}$ [20]), near the zone corner, a $58 \mathrm{eV}$ photon energy detects the bands at $k_{z}=3.8 \pi / c$ and the $45 \mathrm{eV}$ photon energy detects the bands at $k_{z}=3.4 \pi / c$. We assume that the used photon energies 58 and $45 \mathrm{eV}$ are nearly close to the high-symmetry points $M$ and $A$, respectively. Figures 1(e) and 1(f) are the EDMs measured using the $p$ - and $s$-polarized lights with a photon energy of $58 \mathrm{eV}$, respectively, along the cut 2 as showed on the FS map. Figures 1(g) and 1(h) are the analogous data to 1(e) and 1(f), but measured with a photon energy of $45 \mathrm{eV}$. Figures 1(i)-1(n) are the second derivatives of 1(c)-1(h), respectively. Using the photon energy $58 \mathrm{eV}$, one could detect the bands at a $k_{z}=4.06 \pi / c$ (close to $\Gamma$ ) and with $45 \mathrm{eV}$ one could detect the bands at a $k_{z}=3.6 \pi / c$ (close to $Z$ ) near the zone center. All the EDMs showed in Fig. 1 are recorded along the $\Gamma-M$ high-symmetry line.

From Figs. 1(c) and 1(d), we could resolve an electronlike band $\beta$, crossing the Fermi level at a momentum vector $k_{F}=$ $0.08 \pm 0.02 \AA^{-1}$ near the $M$ point, while near the $A$ point, it crosses $E_{F}$ at a $k_{F}=0.1 \pm 0.02 \AA^{-1}$. Thus the band $\beta$ shows no $k_{z}$ dispersion within error bars in going from $M$ to $A$. In Figs. 1(e)-1(h), we could resolve three holelike bands, $\alpha_{1}, \alpha_{2}$, and $\alpha_{3}$, at the high-symmetry points $\Gamma$ and $Z$. A Fermi vector $k_{F}=0.22 \pm 0.02 \AA^{-1}$ for $\alpha_{3}$ band is estimated by comparing the experimental data with DFT calculations both at $\Gamma$ and $Z$. With the help of first-principles calculations and polarizationdependent selection rules, we ascribe $x y$ orbital character to the outermost hole pocket $\alpha_{3}$, while $x z / y z$ character to the hole pockets $\alpha_{1}, \alpha_{2}$ and the electron pocket $\beta$. Generally, the photoemission cross section is low for the inplane orbitals $(x y)$, hence the band $\alpha_{3}$ is scarcely resolved both at $\Gamma$ and $Z$ [see Figs. 1(e) and $1(\mathrm{~g})$ ]. The band $\alpha_{2}$ disperses strongly towards $E_{F}$ but does not cross it, forming a van Hove singularity near the Fermi level at the $\Gamma$ point, consistent with the iron pnictide superconductors [54-58]. On the other hand, near the $Z$ point it crosses $E_{F}$ at $k_{F}=0.11 \pm 0.02 \AA^{-1}$ and thus the band $\alpha_{2}$ is showing a finite $k_{z}$ dispersion. The band $\alpha_{1}$ is always below the Fermi level at both $\Gamma$ and $Z$, hence does not contribute to the Fermi surface.

In order to match the DFT band structure with the experimental dispersions [see Figs. 1(o) and 1(p)] the bands $\alpha_{1}, \alpha_{2}$, and $\alpha_{3}$ are renormalized by a factor of $2,2.6$, and 4.6 and shifted by $-78,-48$, and $-3 \mathrm{meV}$, respectively, near the $\Gamma$ point. Similarly, near the $Z$ point, $\alpha_{1}, \alpha_{2}$, and $\alpha_{3}$ are renormalized by a factor of $0.65,2$, and 4.6 , and shifted by $+170,-117$, and $+8 \mathrm{meV}$, respectively. The band $\beta$ is renormalized by a factor of 2.5 and 0.65 and shifted by +65 and $+327 \mathrm{meV}$ at $M$ and $A$, respectively. Here negative energies represent band shifting towards higher binding energies and positive energies represent band shifting towards lower binding energies.
Figure 2 shows ARPES data from an electron doped $\mathrm{Fe}_{0.9} \mathrm{Ni}_{0.1} \mathrm{Te}_{0.5} \mathrm{Se}_{0.5}$ compound. Figure 2(a) depicts the Fermi surface map measured using $s$-polarized light with an excitation energy $h v=81 \mathrm{eV}$, extracted by integrating over an energy window of $10 \mathrm{meV}$ centered at $E_{F}$, in which hole pockets at the zone center and electron pockets at the zone corner are seen. The data were recorded at a sample temperature of $50 \mathrm{~K}$. Figures 2(b) and 2(c) show the EDMs taken along the cut 1 as shown on the FS map, measured using the $s$-polarized light at $M$ and $A$, respectively. Figures 2(d) and 2(e) show the EDMs taken along the cut 2 as shown on the FS map measured using the $p$-and $s$-polarized lights at $\Gamma$, respectively. Figures $2(\mathrm{f})$ and $2(\mathrm{~g})$ are the analogous data to 2(d) and 2(e), but measured at $Z$ point. Figures $2(\mathrm{~h})-2(\mathrm{~m})$ are the second derivatives of $2(\mathrm{~b})-2(\mathrm{~g})$, respectively. All the EDMs shown in Fig. 2 are recorded along the $\Gamma-M$ high-symmetry line.

From Figs. 2(b) and 2(c), we could resolve an electronlike band $\beta$, crossing the Fermi level at a momentum vector $k_{F}=0.23 \pm 0.02 \AA^{-1}$ near the $M$ point, while near the $A$ point, it crosses $E_{F}$ at $k_{F}=0.25 \pm 0.02 \AA^{-1}$. Thus the band $\beta$ shows no $k_{z}$ dispersion within error bars in going from $M$ to $A$. From Figs. 2(d)-2(g), we could resolve only two holelike bands, $\alpha_{1}$ and $\alpha_{2}$, at both the high-symmetry points $\Gamma$ and $Z$. We again ascribe $x z / y z$ character to the bands $\alpha_{1}, \alpha_{2}$, and $\beta$. Here, the bands $\alpha_{1}$ and $\alpha_{2}$ do not cross the Fermi level near $\Gamma$, while only $\alpha_{2}$ crosses $E_{F}$ with a negligible Fermi vector $k_{F}=0.04 \pm 0.01 \AA^{-1}$ near $Z$. Next, we show ARPES measurements performed to reveal information on the $k_{z}$-dependent electronic structure near the zone corner. For this, photon energy-dependent ARPES spectra were recorded along $k_{y}$, with photon energies ranging from $h v=45$ to $81 \mathrm{eV}$ in steps of $3 \mathrm{eV}$. The data were recorded using the $s$-polarized light along the $\Gamma-M$ high-symmetry line. Figure 2(n) depicts the Fermi surface map in the $k_{y}-k_{z}$ plane measured for $\mathrm{Fe}_{0.9} \mathrm{Ni}_{0.1} \mathrm{Te}_{0.5} \mathrm{Se}_{0.5}$, extracted by integrating over a window range of $15 \mathrm{meV}$ centered at $E_{F}$. Figure 2(o) shows a stack-plot of momentum distribution curves as a function of photon energy, fitted with a pair of Lorentzian functions. The peak positions of the $\beta$ band near the Fermi level extracted from the fits are shown by the black circles on the FS map [see Fig. 2(n)], again suggesting that the electron pocket shows a weak $k_{z}$ dispersion.

In Fig. 3, the red and blue curves are the band dispersions from $\mathrm{FeTe}_{0.5} \mathrm{Se}_{0.5}$ and $\mathrm{Fe}_{0.9} \mathrm{Ni}_{0.1} \mathrm{Te}_{0.5} \mathrm{Se}_{0.5}$, respectively, resulted from a fit to the MDCs using a pair of Lorentzian functions at $\Gamma$ for the band $\alpha_{1}$ [Fig. 3(a)] and for $\alpha_{2}$ [Fig. 3(b)]. Similarly, the band dispersions are shown at $Z$ for $\alpha_{1}$ [Fig. 3(c)] and for $\alpha_{2}$ [Fig. 3(d)]. Panels 3(e) and 3(f) depict the band dispersions from $M$ and $A$, respectively. In Figs. 3(a)-3(f), the solid curves are parabolic fits to the band dispersions from which we could extract the effective masses $\left(m^{*}\right)$. Figure 3(g) shows the DFT-LDA band structure (black curves) with overlaid experimental band dispersions along the $\Gamma-M$ high-symmetry line. Similarly, Fig. 3(h) shows the DFT-LDA band structure with overlaid experimental band dispersions along the $Z$ - $A$ high-symmetry line. 

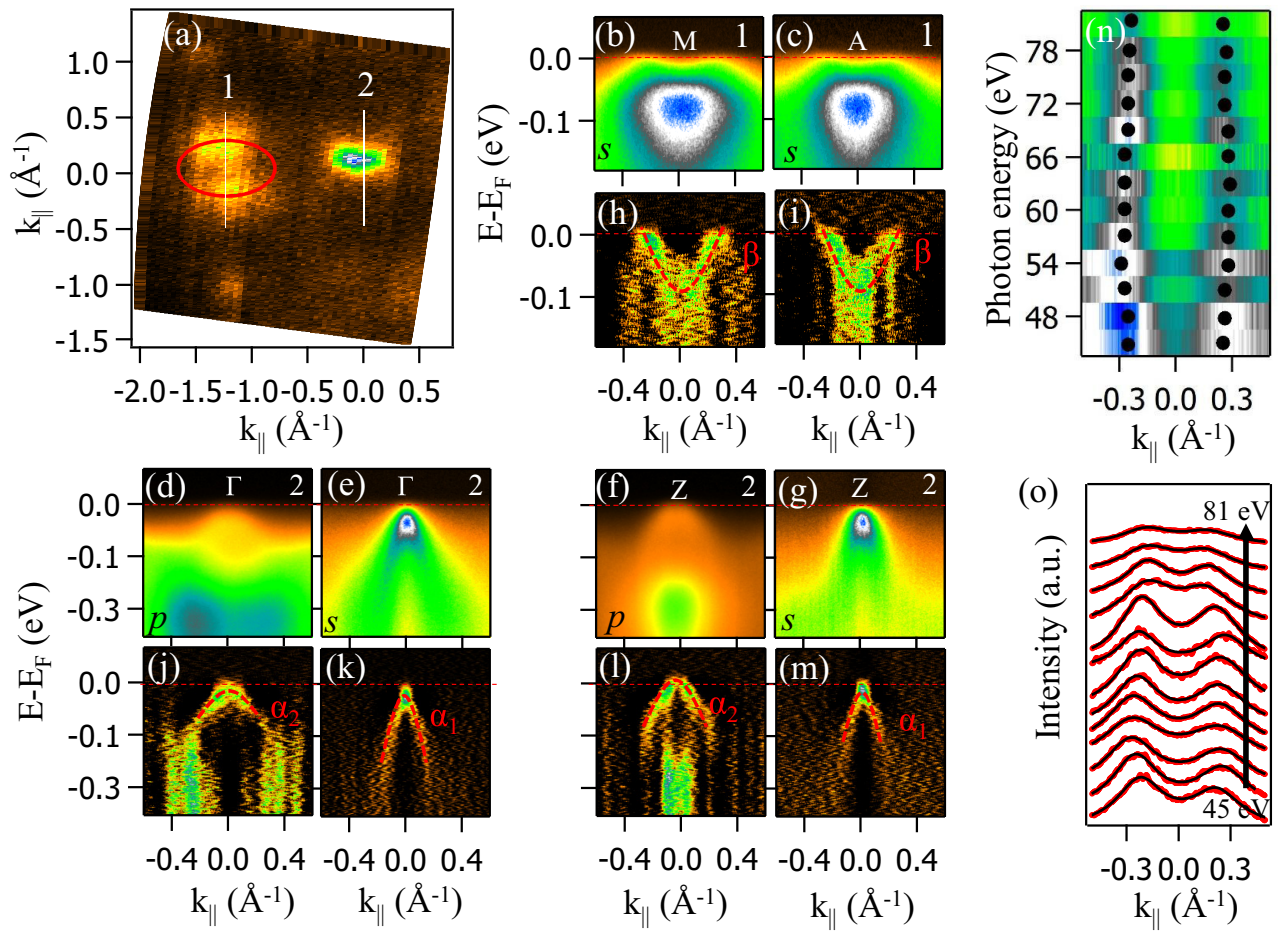

FIG. 2. ARPES spectra of $\mathrm{Fe}_{0.9} \mathrm{Ni}_{0.1} \mathrm{Te}_{0.5} \mathrm{Se}_{0.5}$. (a) is the Fermi surface map measured using $s$-polarized light with a photon energy $h v=81 \mathrm{eV}$. (b) and (c) show the energy distribution maps (EDMs) taken close to the high-symmetry points $M$ and $A$ (see the text), respectively, measured using $s$-polarized light along the cut 1 as shown on the FS map. (d) and (e) are EDMs taken near $\Gamma$ measured using $p$ - and $s$-polarized lights, respectively, along the cut 2 as shown on the FS map. (f) and (g) are analogous data to (d) and (e), respectively, but taken near the $Z$ point. (h)-(m) are the second derivatives of (b)-(g). (n) is the Fermi surface map in the $k_{y}-k_{z}$ plane at the zone corner. The black circles are overlaid on the FS map representing the peak positions of the $\beta$ band. (o) shows a stack-plot of momentum distribution curves (MDCs) sampling different $k_{z}$ (red curves), together with results of a fit using a pair of Lorentzian functions. The red colored contour in (a) is guide to the eye schematically representing the Fermi sheet and the dashed curves in $(\mathrm{h})-(\mathrm{m})$ are guides to the eye schematically representing the band dispersions. We have normalized the EDMs by higher orders monochromator above the Fermi level.

\section{DISCUSSIONS}

We could resolve three hole pockets, $\alpha_{1}, \alpha_{2}$, and $\alpha_{3}$, around $\Gamma$ and $Z$ and an electron pocket $\beta$ around $M$ and $A$ from the $\mathrm{FeTe}_{0.5} \mathrm{Se}_{0.5}$ superconductor (see Fig. 1). This observation is consistent with the ARPES reports on similar compounds [38,39,41-43]. Effective mass enhancements are estimated for this compound upon employing parabolic fits (see Fig. 3) to the experimental band dispersions $\left(m^{*}\right)$ and the DFT band structure $\left(m_{b}\right)$. We have calculated a mass
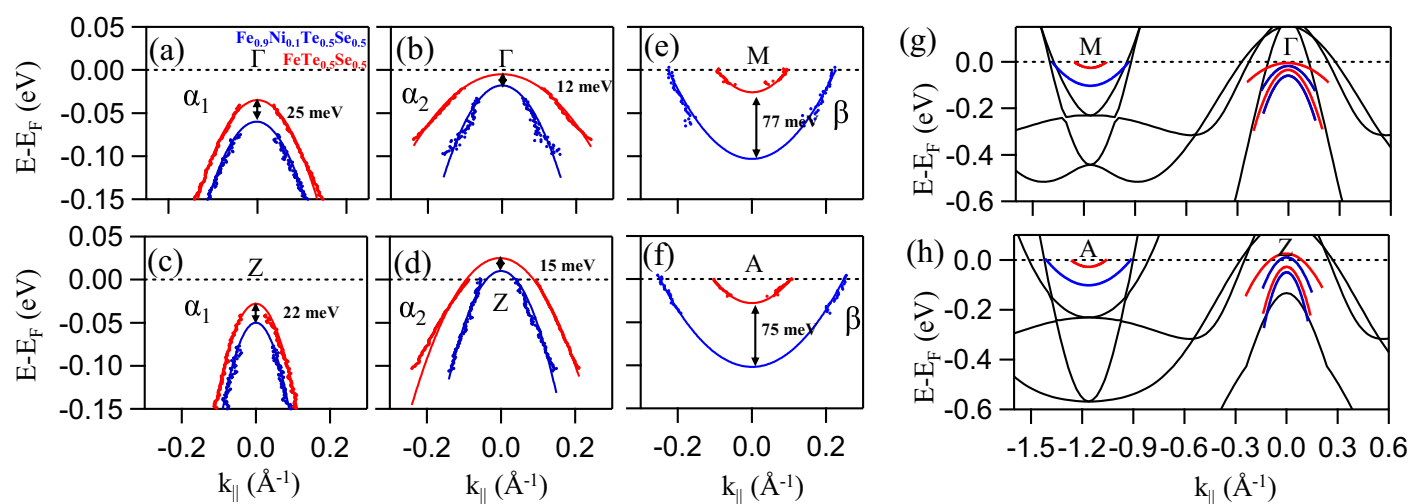

FIG. 3. In the figure, the red and blue curves are the band dispersions from $\mathrm{FeTe}_{0.5} \mathrm{Se}_{0.5}$ and $\mathrm{Fe}_{0.9} \mathrm{Ni}_{0.1} \mathrm{Te}_{0.5} \mathrm{Se}_{0.5}$, respectively, resulted from a fit to the MDCs using a pair of Lorentzian functions at $\Gamma$ for the band $\alpha_{1}$ (a) and for $\alpha_{2}$ (b). Similarly, the band dispersions are shown at $Z$ for $\alpha_{1}$ (c) and for $\alpha_{2}$ (d). (e) and (f) depict the band dispersions from $M$ and $A$, respectively. (g) shows the DFT-LDA (black curves) band structure overlaid with the experimental band dispersions along the $\Gamma-M$ high-symmetry line. Similarly, (h) shows the band structure overlaid with the experimental band dispersions along the $Z$ - $A$ high-symmetry line. 
renormalization factor $\left(m^{*} / m_{b}\right)$ for the bands $\alpha_{1}, \alpha_{2}$, and $\alpha_{3}$ of $1.77 \pm 0.04,2.23 \pm 0.05$, and $4.76 \pm 0.20$, respectively, near $\Gamma$ and $0.62 \pm 0.01,1.74 \pm 0.22$, and $4.76 \pm 0.20$, respectively, near $Z$. Similarly, the band $\beta$ near $M$ and $A$ shows a mass renormalization factor of $1.78 \pm 0.16$ and $0.66 \pm 0.03$, respectively. The mass renormalization values extracted from the parabola fits are very much close to the values extracted independently by scaling the DFT band structure, as discussed in the previous section. Here we can notice that the mass renormalization factors are relatively smaller at the $Z$ point compared to the $\Gamma$ point for the bands $\alpha_{1}, \alpha_{2}$ and $\beta$, which further suggests $k_{z}$-dependent correlations in these compounds. On the whole, our findings on the band dependent mass renormalization factors ranging from $1.7-5$ observed in the $\Gamma-M$ plane are in very good agreement with previous reports [39-43,59,60], while contradicting to the mass renormalization factor of 17 reported in Ref. [38]. However, Ref. [61] has reported a huge mass renormalization at the Lifshitz transition in the ferropnictides, but nevertheless at higher binding energies the mass renormalization is not far from the present values.

Coming to the main results of this paper, we could resolve only two hole pockets near $\Gamma$ and $Z$ in the case of electron doped $\mathrm{Fe}_{0.9} \mathrm{Ni}_{0.1} \mathrm{Te}_{0.5} \mathrm{Se}_{0.5}$ compound. Recently, a similar observation has been made on a non-Fe-stoichiometric $\mathrm{Fe}_{1.068} \mathrm{Te}_{0.54} \mathrm{Se}_{0.36}$ compound in which case also only two hole pockets are resolved [60]. Note here that the former compound is a nonsuperconductor, while the latter one is a superconductor. However, from both compounds, the third hole pocket $\left(\alpha_{3}\right)$ mainly composed of the $x y$ orbital is unable to detect experimentally. From this, we can understand that $x y$ hole pocket is very sensitive to the system's stoichiometry and the impurity scattering. Because $\alpha_{3}$ has low scattering cross section in ARPES measurements and has higher-band renormalization compared to the other hole pockets, which further broadens the spectral function, it could be that we are unable to detect this band from our ARPES studies when the impurities are added. However, theoretically we do not exclude its presence in $\mathrm{Fe}_{0.9} \mathrm{Ni}_{0.1} \mathrm{Te}_{0.5} \mathrm{Se}_{0.5}$. Importantly, we noticed that, upon electron doping, in $\mathrm{Fe}_{0.9} \mathrm{Ni}_{0.1} \mathrm{Te}_{0.5} \mathrm{Se}_{0.5}$ the chemical potential shifts in accord with a nonrigid band model, unlike in 122-type and 111-type compounds where the impurity substitution shifts the bands in a rigid band model $[17,18,24]$. It is important to note here that without inducing correlations to the system with impurity substitution, the charge doping leads to a rigid band model irrespective of the number of excess carriers contributing to the conduction band $[17,24]$. Therefore the observed nonrigid band type chemical potential shift in the studied compound is directly related to the induced correlation effects (discussed below) with the substituted impurity potential [18].

From Figs. 3(a)-3(d), it is clear that the top of holelike bands near $\Gamma$ shifts towards higher binding energy almost by an average of $23 \mathrm{meV}$ and near $Z$ it is of $13 \mathrm{meV}$ upon the electron doping. On the other hand, near the zone corner [see Figs. 3(e) and 3(f)], we noticed that the bottom of electronlike band shifts towards the higher binding energy almost by an average of $76 \mathrm{meV}$. These details indicate that the Ni doping is leading to an effective electron doping. Thus the size of the hole pockets got shrunk and the electron pockets got increased, but unequally. That means the decrease in the hole pocket size is relatively low compared to the increase in the electron pocket. This observation is not supporting the experimental [19-23,25,30,31] and theoretical [17,24,62] reports on weakly correlated 122-type and 111-type compounds, which suggests that in going from the substitution of $\mathrm{Co}$ to $\mathrm{Ni}$ to $\mathrm{Cu}$ to $\mathrm{Zn}$ in the place of Fe, the volume of the Fermi sheets increases and decreases, which is qualitatively consistent with the rigid-band model.

Interestingly, the mass renormalizations are reduced near the zone center and are increased near the zone corner upon the impurity substitution. More precisely, the effective mass ( $m_{\mathrm{Ni}}^{*}$ ) got reduced in $\mathrm{Fe}_{0.9} \mathrm{Ni}_{0.1} \mathrm{Te}_{0.5} \mathrm{Se}_{0.5}$ as compared to the $\mathrm{FeTe}_{0.5} \mathrm{Se}_{0.5}$ superconductor $\left(m^{*}\right)$ by a factor $\left(m^{*} / m_{\mathrm{Ni}}^{*}\right)$ of $1.21 \pm 0.02$ and $2.54 \pm 0.26$ for $\alpha_{1}$ and $\alpha_{2}$, respectively, near $\Gamma$, while $1.06 \pm 0.03$ and $2.24 \pm 0.03$ for $\alpha_{1}$ and $\alpha_{2}$, respectively near $Z$. On the other hand, the effective mass $\left(m_{\mathrm{Ni}}^{*} / m^{*}\right)$ got increased by a factor of $1.4 \pm 0.1$ and $1.5 \pm 0.1$ at $M$ and $A$, respectively. Here, we can notice that the estimated mass enhancements with $\mathrm{Ni}$ substitution are almost same within error bars both at $\Gamma$ and $Z$. This clearly demonstrates that $\mathrm{Ni}$ doping does not induce additional $k_{z}$ dependent correlations. In principle, the impurity substitution could affect the electronic structure of the host by three different ways: (1) the crystal field splitting, (2) the average scattering potential, which is generally determined by the onsite Coulomb energy $(U)$, and (3) the electron-impurity scattering. In the present case, $\mathrm{Fe}$ is partially replaced by $\mathrm{Ni}$ which has a similar ionic size as Fe. Therefore the crystal field splitting in the electronic structure should be negligible [45]. Similarly, the increase of onsite $U$ when going from $\mathrm{Fe}$ to $\mathrm{Ni}$ is small [63], which probably also indicates that this effect does not determine the observed effective mass changes. On the other hand, Refs. [18] and [62] suggest an enhanced complex self-energy with the impurity substitution due to the electron-impurity scattering, which consequently enhances the real part of the self-energy and thus the effective mass. The increased effective mass for the electron pockets with $\mathrm{Ni}$ substitution is consistent with Ref. [62], however, the decreased effective mass for the hole pockets is in contrast and is further demonstrating the complexity in the microscopic understanding of the impurity substitution effect on the electronic structure of the iron-based compounds [18]. Moreover, there are several ARPES reports on this issue in the case of 122-type compounds, which further demonstrate the complexity of understanding this problem [19,23,31]. For instance, Refs. [19] and [31] show decreased electronic correlations, while Ref. [23] shows unchanged electronic correlations upon the impurity substitution.

Finally, in the $\mathrm{FeTe}_{0.5} \mathrm{Se}_{0.5}$ superconductor, we could notice hole Fermi sheets at the $\Gamma$ point and electron Fermi sheets at the $M$ point, hence there the low-energy interband scattering between the hole and the electron pockets (see Fig. 1) is possible. On the other hand, in $\mathrm{Fe}_{0.9} \mathrm{Ni}_{0.1} \mathrm{Te}_{0.5} \mathrm{Se}_{0.5}$ near the $\Gamma$ point, the hole pockets are completely filled by the electrons with Ni substitution and are stretched below the Fermi level (see Fig. 2), thus the low-energy interband scattering between hole and electron pockets, which is believed to be important for high- $T_{c}$ superconductivity in these compounds [14,64,65] is totally suppressed. This could be a natural explanation on, why the $\mathrm{Ni}$ substitution does turn the compound from a superconductor to a nonsuperconductor. Before closing 
this section, we would like to explicitly mention that the temperature difference between the data of $\mathrm{FeTe}_{0.5} \mathrm{Se}_{0.5}$ and $\mathrm{Fe}_{0.9} \mathrm{Ni}_{0.1} \mathrm{Te}_{0.5} \mathrm{Se}_{0.5}$ does not affect the conclusions of this paper, because the estimated band dispersions (see Fig. 3) based on analysis of the momentum distribution curves does not depend much on the measured sample temperature.

\section{CONCLUSIONS}

In conclusion, we have studied the effect of impurity potential on the electronic structure of $\mathrm{FeTe}_{0.5} \mathrm{Se}_{0.5}$ superconductor by substituting $10 \%$ of $\mathrm{Ni}$ for $\mathrm{Fe}$. We could resolve three hole pockets near the zone center and an electron pocket near the zone corner in the case of $\mathrm{FeTe}_{0.5} \mathrm{Se}_{0.5}$, whereas only two hole pockets near the center and an electron pocket near the corner of the Brillouin zone are resolved in the case of $\mathrm{Fe}_{0.9} \mathrm{Ni}_{0.1} \mathrm{Te}_{0.5} \mathrm{Se}_{0.5}$, suggesting that the third hole pocket having predominately $x y$ orbital character is very sensitive to the impurity scattering. We observe a decrease in the size of the hole pockets and an increase in the size of the electron pockets with Ni substitution, suggesting an effective electron doping. However, the change in the size of the electron and hole pockets is not consistent with the rigid band model. We further noticed that the effective mass of the hole pockets is reduced near the zone center and the effective mass of the electron pockets is increased near the zone corner in $\mathrm{Fe}_{0.9} \mathrm{Ni}_{0.1} \mathrm{Te}_{0.5} \mathrm{Se}_{0.5}$ when compared to $\mathrm{FeTe}_{0.5} \mathrm{Se}_{0.5}$. We suggest that the peculiarity of the nonrigid band changes of the chemical potential with $\mathrm{Ni}$ substitution is directly related to reduced correlations at the zone center and increased correlations at the zone corner. We could notice the interband scattering between the hole and electron Fermi sheets in the $\mathrm{FeTe}_{0.5} \mathrm{Se}_{0.5}$ superconductor, whereas in nonsuperconducting $\mathrm{Fe}_{0.9} \mathrm{Ni}_{0.1} \mathrm{Te}_{0.5} \mathrm{Se}_{0.5}$ compound the interband band scattering is suppressed as the hole pockets near the $\Gamma$ point are completely filled by the added electrons, suggesting that the Fermi surface topology is essential for high- $T_{c}$ superconductivity in these compounds.

\section{ACKNOWLEDGMENTS}

T.S. acknowledges support by the Department of Science and Technology (DST) through INSPIRE-Faculty program (Grant number: IFA14 PH-86). T.S. acknowledges greatly the travel support given by IFW Dresden for part of the measurements. J.F. and B.B. acknowledge support by the German Research Foundation (DFG) through the priority program SPP1458. V.P.S.A. and P.K.M. acknowledge the financial support from the Department of Atomic Energy (DAE) through the DAE-SRC outstanding researcher scheme.
[1] Y. Kamihara, T. Watanabe, M. Hirano, and H. Hosono, J. Am. Chem. Soc. 130, 3296 (2008).

[2] M. Rotter, M. Tegel, D. Johrendt, I. Schellenberg, W. Hermes, and R. Pöttgen, Phys. Rev. B 78, 020503(R) (2008).

[3] H. S. Jeevan, Z. Hossain, D. Kasinathan, H. Rosner, C. Geibel, and P. Gegenwart, Phys. Rev. B 78, 052502 (2008).

[4] J. H. Tapp, Z. Tang, B. Lv, K. Sasmal, B. Lorenz, P. C. W. Chu, and A. M. Guloy, Phys. Rev. B 78, 060505 (2008).

[5] C. Chu, F. Chen, M. Gooch, A. Guloy, B. Lorenz, B. Lv, K. Sasmal, Z. Tang, J. Tapp, and Y. Xue, Physica C 469, 326 (2009).

[6] Y. Mizuguchi, F. Tomioka, S. Tsuda, T. Yamaguchi, and Y. Takano, Physica C: Superconductivity 469, 1027 (2009).

[7] X. Wang, Q. Liu, Y. Lv, W. Gao, L. Yang, R. Yu, F. Li, and C. Jin, Solid State Commun. 148, 538 (2008).

[8] F.-C. Hsu, J.-Y. Luo, K.-W. Yeh, T.-K. Chen, T.-W. Huang, P. M. Wu, Y.-C. Lee, Y.-L. Huang, Y.-Y. Chu, D.-C. Yan, and M.-K. Wu, Proc. Natl. Acad. Sci. U. S. A. 105, 14262 (2008).

[9] M. Rotter, M. Tegel, and D. Johrendt, Phys. Rev. Lett. 101, 107006 (2008).

[10] H. S. Jeevan, Z. Hossain, D. Kasinathan, H. Rosner, C. Geibel, and P. Gegenwart, Phys. Rev. B 78, 092406 (2008).

[11] M. A. Tanatar, N. Ni, C. Martin, R. T. Gordon, H. Kim, V. G. Kogan, G. D. Samolyuk, S. L. Bud'ko, P. C. Canfield, and R. Prozorov, Phys. Rev. B 79, 094507 (2009).

[12] F. Massee, S. de Jong, Y. Huang, J. Kaas, E. van Heumen, J. B. Goedkoop, and M. S. Golden, Phys. Rev. B 80, 140507 (2009).

[13] I. I. Mazin, M. D. Johannes, L. Boeri, K. Koepernik, and D. J. Singh, Phys. Rev. B 78, 085104 (2008).
[14] K. Kuroki, S. Onari, R. Arita, H. Usui, Y. Tanaka, H. Kontani, and H. Aoki, Phys. Rev. Lett. 101, 087004 (2008).

[15] L. Zhang and D. J. Singh, Phys. Rev. B 80, 214530 (2009).

[16] H. Wadati, I. Elfimov, and G. A. Sawatzky, Phys. Rev. Lett. 105, 157004 (2010).

[17] K. Nakamura, R. Arita, and H. Ikeda, Phys. Rev. B 83, 144512 (2011).

[18] T. Berlijn, C.-H. Lin, W. Garber, and W. Ku, Phys. Rev. Lett. 108, 207003 (2012).

[19] V. Brouet, M. Marsi, B. Mansart, A. Nicolaou, A. TalebIbrahimi, P. Le Fevre, F. Bertran, F. Rullier-Albenque, A. Forget, and D. Colson, Phys. Rev. B 80, 165115 (2009).

[20] S. Thirupathaiah, S. de Jong, R. Ovsyannikov, H. A. Dürr, A. Varykhalov, R. Follath, Y. Huang, R. Huisman, M. S. Golden, Y.-Z. Zhang, H. O. Jeschke, R. Valentí, A. Erb, A. Gloskovskii, and J. Fink, Phys. Rev. B 81, 104512 (2010).

[21] Y. Sekiba, T. Sato, K. Nakayama, K. Terashima, P. Richard, J. H. Bowen, H. Ding, Y.-M. Xu, L. J. Li, G. H. Cao, Z.-A. Xu, and T. Takahashi, New J. Phys. 11, 025020 (2009).

[22] M. Neupane, P. Richard, Y.-M. Xu, K. Nakayama, T. Sato, T. Takahashi, A. V. Federov, G. Xu, X. Dai, Z. Fang, Z. Wang, G.-F. Chen, N.-L. Wang, H.-H. Wen, and H. Ding, Phys. Rev. B 83, 094522 (2011).

[23] R. S. Dhaka, Y. Lee, V. K. Anand, D. C. Johnston, B. N. Harmon, and A. Kaminski, Phys. Rev. B 87, 214516 (2013).

[24] S. Ideta, T. Yoshida, I. Nishi, A. Fujimori, Y. Kotani, K. Ono, Y. Nakashima, S. Yamaichi, T. Sasagawa, M. Nakajima, K. Kihou, Y. Tomioka, C. H. Lee, A. Iyo, H. Eisaki, T. Ito, S. Uchida, and R. Arita, Phys. Rev. Lett. 110, 107007 (2013). 
[25] M. Merz, F. Eilers, T. Wolf, P. Nagel, H. v. Löhneysen, and S. Schuppler, Phys. Rev. B 86, 104503 (2012).

[26] J. P. Clancy, B. D. Gaulin, and A. S. Sefat, Phys. Rev. B 85, 054115 (2012).

[27] Y. Texier, Y. Laplace, P. Mendels, J. T. Park, G. Friemel, D. L. Sun, D. S. Inosov, C. T. Lin, and J. Bobroff, Europhys. Lett. 99, 17002 (2012).

[28] E. D. L. Rienks, T. Wolf, K. Koepernik, I. Avigo, P. Hlawenka, C. Lupulescu, T. Arion, F. Roth, W. Eberhardt, U. Bovensiepen, and J. Fink, Europhys. Lett. 103, 47004 (2013).

[29] S. Thirupathaiah, D. V. Evtushinsky, J. Maletz, V. B. Zabolotnyy, A. A. Kordyuk, T. K. Kim, S. Wurmehl, M. Roslova, I. Morozov, B. Büchner, and S. V. Borisenko, Phys. Rev. B 86, 214508 (2012).

[30] S. T. Cui, S. Kong, S. L. Ju, P. Wu, A. F. Wang, X. G. Luo, X. H. Chen, G. B. Zhang, and Z. Sun, Phys. Rev. B 88, 245112 (2013).

[31] L. Y. Xing, H. Miao, X. C. Wang, J. Ma, Q. Q. Liu, Z. Deng, H. Ding, and C. Q. Jin, J. Phys.: Condens. Matter 26, 435703 (2014).

[32] A. J. Williams, T. M. McQueen, V. Ksenofontov, C. Felser, and R. J. Cava, J. Phys.: Condens. Matter 21, 305701 (2009).

[33] R. Shipra, H. Takeya, K. Hirata, and A. Sundaresan, Physica C: Superconductivity 470, 528 (2010).

[34] T.-W. Huang, T.-K. Chen, K.-W. Yeh, C.-T. Ke, C. L. Chen, Y.-L. Huang, F.-C. Hsu, M.-K. Wu, P. M. Wu, M. Avdeev, and A. J. Studer, Phys. Rev. B 82, 104502 (2010).

[35] J. Wang, R. Zhong, S. Li, Y. Gan, Z. Xu, C. Zhang, T. Ozaki, M. Matsuda, Y. Zhao, Q. Li, G. Xu, G. Gu, J. M. Tranquada, R. J. Birgeneau, and J. Wen, Phys. Rev. B 91, 014501 (2015).

[36] E. L. Thomas, W. Wong-Ng, D. Phelan, and J. N. Millican, J. Appl. Phys. 105, 073906 (2009).

[37] J. Wen, S. Li, Z. Xu, C. Zhang, M. Matsuda, O. Sobolev, J. T. Park, A. D. Christianson, E. Bourret-Courchesne, Q. Li, G. Gu, D.-H. Lee, J. M. Tranquada, G. Xu, and R. J. Birgeneau, Phys. Rev. B 88, 144509 (2013).

[38] A. Tamai, A. Y. Ganin, E. Rozbicki, J. Bacsa, W. Meevasana, P. D. C. King, M. Caffio, R. Schaub, S. Margadonna, K. Prassides, M. J. Rosseinsky, and F. Baumberger, Phys. Rev. Lett. 104, 097002 (2010).

[39] A. Yamasaki, Y. Matsui, S. Imada, K. Takase, H. Azuma, T. Muro, Y. Kato, A. Higashiya, A. Sekiyama, S. Suga, M. Yabashi, K. Tamasaku, T. Ishikawa, K. Terashima, H. Kobori, A. Sugimura, N. Umeyama, H. Sato, Y. Hara, N. Miyagawa, and S. I. Ikeda, Phys. Rev. B 82, 184511 (2010).

[40] J. Maletz, V. B. Zabolotnyy, D. V. Evtushinsky, S. Thirupathaiah, A. U. B. Wolter, L. Harnagea, A. N. Yaresko, A. N. Vasiliev, D. A. Chareev, A. E. Böhmer, F. Hardy, T. Wolf, C. Meingast, E. D. L. Rienks, B. Büchner, and S. V. Borisenko, Phys. Rev. B 89, 220506 (2014).

[41] L. C. C. Ambolode, K. Okazaki, M. Horio, H. Suzuki, L. Liu, S. Ideta, T. Yoshida, T. Mikami, T. Kakeshita, S. Uchida, K. Ono, H. Kumigashira, M. Hashimoto, D.-H. Lu, Z.-X. Shen, and A. Fujimori, Phys. Rev. B 92, 035104 (2015).

[42] P. Zhang, T. Qian, P. Richard, X. P. Wang, H. Miao, B. Q. Lv, B. B. Fu, T. Wolf, C. Meingast, X. X. Wu, Z. Q. Wang, J. P. Hu, and H. Ding, Phys. Rev. B 91, 214503 (2015).

[43] M. Yi, Z.-K. Liu, Y. Zhang, R. Yu, J. X. Zhu, J. J. Lee, R. G. Moore, F. T. Schmitt, W. Li, S. C. Riggs, J. H. Chu, B. Lv, J. Hu,
M. Hashimoto, S. K. Mo, Z. Hussain, Z. Q. Mao, C. W. Chu, I. R. Fisher, Q. Si, Z. X. Shen, and D. H. Lu, Nat. Commun. 6, 7777 (2015).

[44] P. K. Maheshwari, R. Jha, B. Gahtori, and V. P. S. Awana, AIP Adv. 5, 097112 (2015).

[45] A. Kumar, R. P. Tandon, and V. P. S. Awana, IEEE Trans. Magn. 48, 4239 (2012).

[46] S. V. Borisenko, Synchrotron Radiation News 25, 6 (2012).

[47] S. V. Borisenko, V. B. Zabolotnyy, D. V. Evtushinsky, T. K. Kim, I. V. Morozov, A. N. Yaresko, A. A. Kordyuk, G. Behr, A. Vasiliev, R. Follath, and B. Büchner, J. Vis. Exp. 68, e50129 (2012).

[48] P. E. Blöchl, Phys. Rev. B 50, 17953 (1994).

[49] G. Kresse and D. Joubert, Phys. Rev. B 59, 1758 (1999).

[50] G. Kresse and J. Hafner, Phys. Rev. B 47, 558 (1993).

[51] G. Kresse and J. Hafner, Phys. Rev. B 49, 14251 (1994).

[52] G. Kresse and J. Furthmüller, Comput. Mater. Sci. 6, 15 (1996).

[53] G. Kresse and J. Furthmüller, Phys. Rev. B 54, 11169 (1996).

[54] C. Liu, T. Kondo, R. M. Fernandes, A. D. Palczewski, E. D. Mun, N. Ni, A. N. Thaler, A. Bostwick, E. Rotenberg, J. Schmalian, S. L. Bud'ko, P. C. Canfield, and A. Kaminski, Nat. Phys. 6, 419 (2010).

[55] V. Zabolotnyy, D. Inosov, B. Evtushinski, A. Koitzsch, A. Kordyuk, J. Park, D. Haug, V. Hinkov, A. Boris, G. Sun, C. Lin, B. Keimer, M. Knupfer, B. Buechner, A. Varykhalov, R. Follath, and S. Borisenko, Nature (London) 457, 569 (2009).

[56] S. V. Borisenko, V. B. Zabolotnyy, D. V. Evtushinsky, T. K. Kim, I. V. Morozov, A. N. Yaresko, A. A. Kordyuk, G. Behr, A. Vasiliev, R. Follath, and B. Büchner, Phys. Rev. Lett. 105, 067002 (2010).

[57] Y. Lubashevsky, E. Lahoud, K. Chashka, D. Podolsky, and A. Kanigel, Nat. Phys. 8, 309 (2012).

[58] S. Thirupathaiah, T. Stürzer, V. B. Zabolotnyy, D. Johrendt, B. B. Büchner, and S. V. Borisenko, Phys. Rev. B 88, 140505(R) (2013).

[59] M. Aichhorn, S. Biermann, T. Miyake, A. Georges, and M. Imada, Phys. Rev. B 82, 064504 (2010).

[60] S. Thirupathaiah, J. Fink, P. K. Maheswari, V. P. S. Awana, E. Slooten, Y. Huang, M. S. Golden, F. Lochner, R. Ovsyannikov, H. Dürr, and I. Eremin, arXiv:1511.00424.

[61] J. Fink, A. Charnukha, E. D. L. Rienks, Z. H. Liu, S. Thirupathaiah, I. Avigo, F. Roth, H. S. Jeevan, P. Gegenwart, M. Roslova, I. Morozov, S. Wurmehl, U. Bovensiepen, S. Borisenko, M. Vojta, and B. Büchner, Phys. Rev. B 92, 201106 (2015).

[62] M. W. Haverkort, I. S. Elfimov, and G. A. Sawatzky, arXiv:1109.4036.

[63] J. Sánchez-Barriga, J. Braun, J. Minár, I. Di Marco, A. Varykhalov, O. Rader, V. Boni, V. Bellini, F. Manghi, H. Ebert, M. I. Katsnelson, A. I. Lichtenstein, O. Eriksson, W. Eberhardt, H. A. Dürr, and J. Fink, Phys. Rev. B 85, 205109 (2012).

[64] I. I. Mazin, D. J. Singh, M. D. Johannes, and M. H. Du, Phys. Rev. Lett. 101, 057003 (2008).

[65] S. Graser, A. F. Kemper, T. A. Maier, H. P. Cheng, P. J. Hirschfeld, and D. J. Scalapino, Phys. Rev. B 81, 214503 (2010). 